VINÍ́IIUS BASSO PRETI

SÉrgio Bruno B. HATSCHBACH ${ }^{2}$ José Clemente LINHARES ${ }^{3}$ JoÃo ANTÔNo GUERRERO ${ }^{3}$

Claudiane LÍGia MINARI ${ }^{3}$ CARLos Afonso MaESTRI ${ }^{3}$ FERNANDA VILLAR FonseCA ${ }^{3}$

\section{Tumor borderline do ovário localizado no canal inguinal: relato de caso}

\author{
A borderline ovarian tumor in inguinal canal: case report
}

Relato de caso

Palavras-chave

Neoplasias ovarianas/diagnóstico Neoplasias ovarianas/cirurgia

Doenças dos anexos

Neoplasias da mama/secundário

Hérnia inguinal

Relatos de casos

Keywords

Ovarian neoplasm/diagnosis Ovarian neoplasm/surgery Hernia, inguinal Breast neoplasms/secondary Adnexal diseases Case reports

\section{Resumo}

Os sintomas do tumor ovariano são inespecíficos e uma forma rara de apresentação é como conteúdo de uma hérnia inguinal. Relatamos o caso de uma paciente de 82 anos, com diagnóstico de câncer de mama e lesão anexial hipoecoica à ecografia. A mesma foi submetida à cirurgia conservadora da mama e à laparotomia, com achado de lesão ovariana sólido-cística no interior do canal inguinal à direita. A análise por congelação foi negativa para malignidade, e o exame anatomopatológico mostrou tratar-se de tumor ovariano borderline.

\section{Abstract}

The symptoms of ovarian tumor are not specific and a rare presentation of the tumor is as the content of an inguinal hernia. We reported a case of an 82-year-old woman, diagnosed with breast cancer and with a concomitant hypoecoic adnexal mass at the ecographic exam. The patient was treated with conservative breast surgery and laparotomy. A cystic-solid ovarian lesion was found inside the right inguinal canal. Frozen-section examination was negative for malignancy, and the anatomopathological analysis revealed a borderline ovarian tumor.
Correspondência:

Vinícius Basso Preti Rua Atillio Bório, 139 - apto. 801 CEP 80050-250 - Curitiba (PR), Brasi E-mail: vbpreti@yahoo.com.br

Recebido

$8 / 9 / 2009$
Trabalho realizado no Serviço de Ginecologia e Mama do Hospital Erasto Gaertner - HEG - Curitiba (PR), Brasil.

'Médico Residente em Cancerologia Cirúrgica do Hospital Erasto Gaertner - HEG - Curitiba (PR), Brasil.

${ }^{2}$ Chefe do Serviço de Ginecologia e Mama do Hospital Erasto Gaertner - HEG - Curitiba (PR), Brasil.

${ }^{3}$ Médicos Titulares do Serviço de Ginecologia e Mama do Hospital Erasto Gaertner - HEG - Curitiba (PR), Brasil. 


\section{Introdução}

O câncer de ovário é o tumor mais letal entre as neoplasias ginecológicas. Estima-se que ocorreram 15 mil mortes no ano de 2006 e que ocorram, aproximadamente, 27 mil casos novos por ano nos Estados Unidos. O tipo mais comum é o de origem epitelial, responsável por mais de $90 \%$ de todos os casos de câncer de ovário ${ }^{1}$. A faixa etária mais frequente de apresentação é entre a sexta e a sétima década de vida, com média de incidência aos 61 anos na América do Norte ${ }^{2}$. Os sintomas são inespecíficos, sendo mais frequentes a dor ou desconforto no hipogástrio e nas fossas ilíacas e a identificação de massa pélvica ao exame físico. A maioria das pacientes sintomáticas apresentase nos estádios III e IV. Algumas pacientes podem ter sintomas raros, dependendo da localização e do tamanho da massa anexial ${ }^{3,4}$.

Lesões malignas raramente se apresentam como conteúdo de saco herniário inguinal. Existem relatos de carcinoma do apêndice, tumor do cólon, lesões císticas benignas anexiais e cistos dermoides como conteúdo de hérnia inguinal ${ }^{5-7}$. A maioria das lesões eram achados operatórios, e poucas apresentavam-se como massas volumosas.

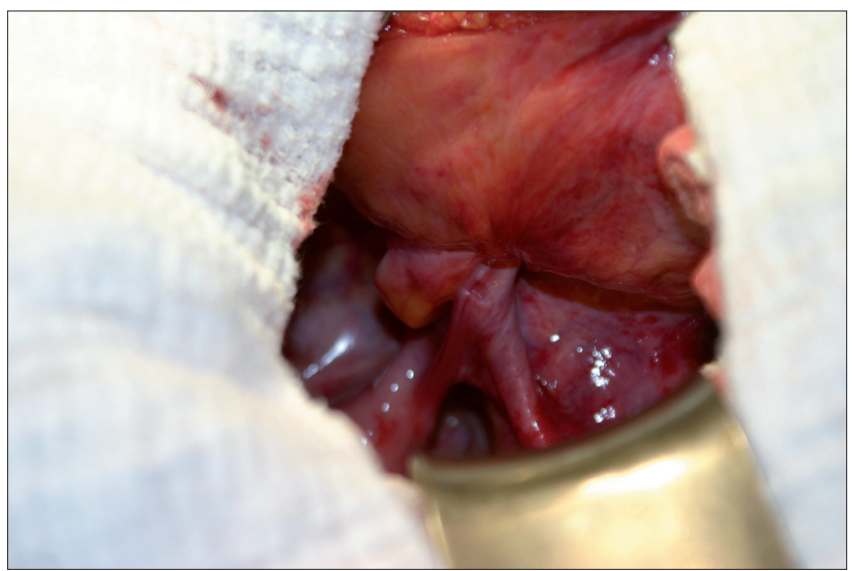

Figura 1 - Achado intraoperatório de tração do ligamento próprio do ovário, tuba uterina e ligamento redondo no canal inguinal profundo.

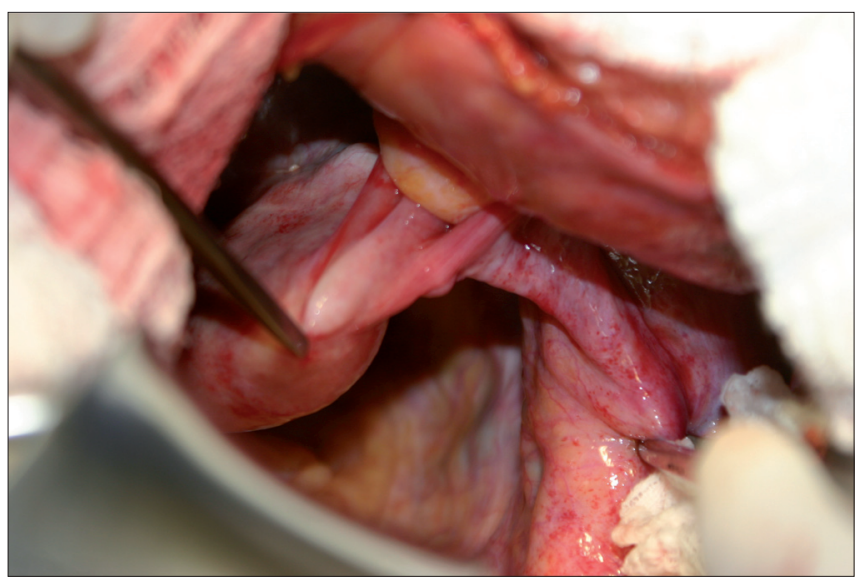

Figura 2 - Tentativa de tração, sem sucesso na retirada da lesão do interior do canal inguinal.
Relatamos um caso, ocorrido em nosso serviço, de paciente portadora de tumor de mama, com achado de tumor de ovário no interior do canal inguinal.

\section{Caso clínico}

Paciente de 82 anos, do sexo feminino, encaminhada com uma mamografia classificada como BIRADS IV-C. Ao exame físico, apresentava nódulo mal delimitado de 1 cm, palpável na mama esquerda, sem linfonodos palpáveis na axila. Ao estadiamento, foi evidenciada, à ecografia de abdome, uma lesão expansiva hipodensa na região anexial direita de 79x60x40 mm, com parte de conteúdo líquido e a maior porção de aspecto hipoecoico, aparentemente encapsulada. Não havia lesões suspeitas de metástase. A paciente foi classificada com estádio clínico I (T1N0M0) e com lesão anexial associada a esclarecer. A dosagem de CA 125 estava dentro dos limites da normalidade. Ao exame físico abdominal, havia tumoração palpável na fossa ilíaca direita, móvel, indolor, de, aproximadamente, $5 \mathrm{~cm}$ - exame dificultado pelo abdome em avental. Devido à idade da paciente, suspeitava-se tratar de uma lesão sincrônica de ovário. O tratamento instituído foi a realização de quadrantectomia e pesquisa de linfonodo sentinela, seguido de laparotomia para estadiamento de tumor de ovário no mesmo tempo cirúrgico.

À inspeção da cavidade, foi observada tração do ligamento próprio do ovário, tuba uterina e ligamento redondo junto ao anel inguinal profundo e palpada massa no interior do canal inguinal (Figura 1). Não foi possível a redução pelo anel inguinal, sendo necessária a abertura do anel inguinal profundo para retirada da lesão (Figuras 2 e 3). $\mathrm{O}$ aspecto era de lesão cística, de $6 \mathrm{~cm}$, do ovário direito (Figura 4). O ovário esquerdo e o útero eram de aspectos normais. Não havia outro achado na inspeção da cavidade. $\mathrm{O}$ material foi encaminhado para exame anatomopatológico de congelação intraoperatória com diagnóstico de lesão negativa para malignidade. Pelo fato

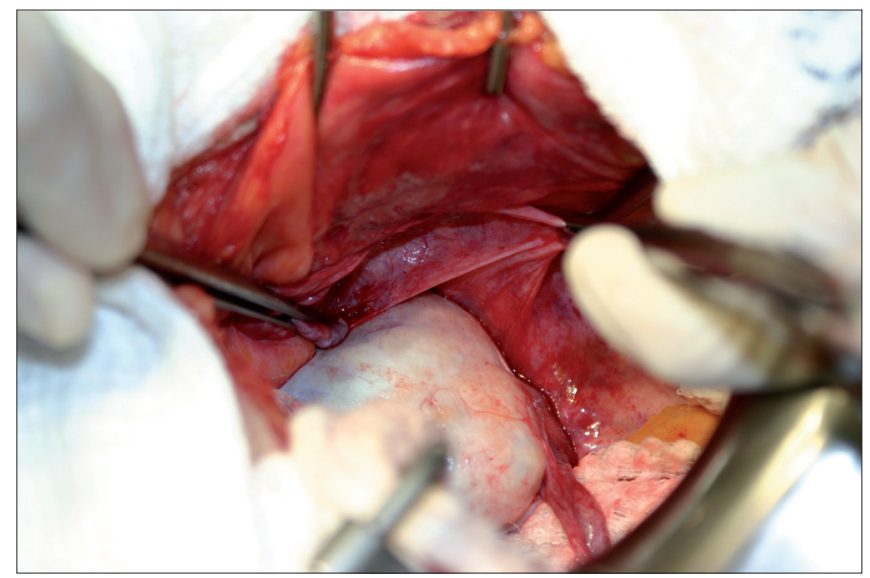

Figura 3 - Redução do tumor de ovário após abertura do anel inguinal profundo. 


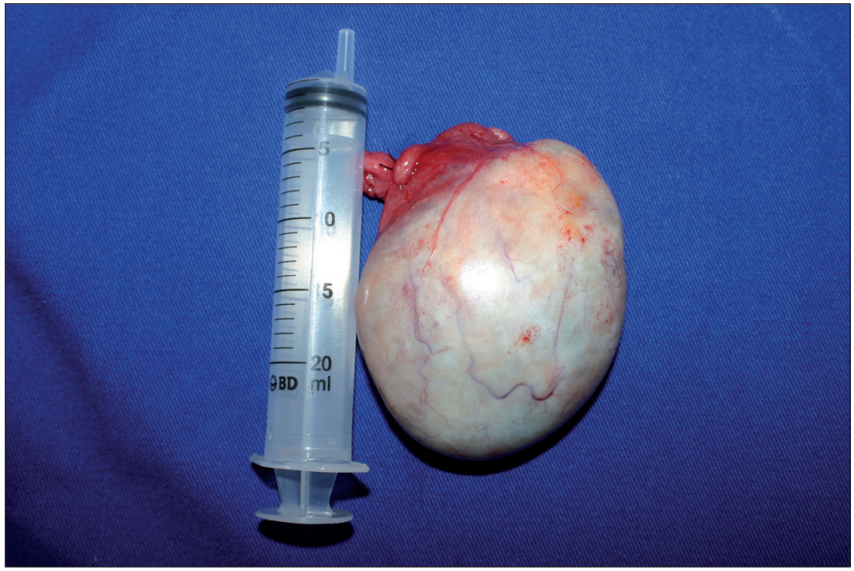

Figura 4 - Aspecto da lesão após a retirada da cavidade abdominal.

de a paciente ser octogenária, foi realizada a ooforectomia contralateral. A hérnia inguinal foi corrigida com sutura da fascia transversalis ao trato iliopúbico e sutura do peritônio, sem utilização de tela de polipropileno. O exame anatomopatológico com hematoxilina-eosina constatou lesão serosa borderline (Figura 5). O seguimento clínico, em relação à lesão ovariana, sem novas intervenções cirúrgicas, foi proposto em virtude da idade e comorbidades da paciente.

O produto da quadrantectomia foi submetido a congelação intraoperatória das margens, as quais estavam livres. Realizada pesquisa do linfonodo sentinela marcado previamente por linfocintilografia. O exame anatomopatológico final da ressecção mamária foi de carcinoma ductal invasivo, grau 2 de Bloom-Richardson, sem evidência de embolia vascular. O linfonodo sentinela mostrou-se livre de neoplasia. A paciente foi submetida a tratamento radioterápico adjuvante. $\mathrm{O}$ receptor estrogênico da mama foi positivo em $90 \%$ das células à imunoistoquímica, sendo iniciada terapia com tamoxifeno.

Dois anos após a operação, a paciente encontrava-se assintomática, com todos os exames de seguimento normais e sem sinais da recidiva da hérnia inguinal.

\section{Discussão}

O conteúdo herniário mais comumente encontrado no interior de hérnia inguinal são segmentos do intestino delgado e cólon, sendo menos frequentes bexiga urinária, anexos uterinos, apêndice cecal e divertículo de $\mathrm{Meckel}^{8}$.

Tumores malignos são extremamente raros, correspondendo a menos de $0,5 \%$ das ressecções de massas inguinais, sendo mais comum o tumor do cólon sigmoide? Outros tumores relatados são lesões mucinosas e recidivas dos pseudomixomas peritoneais ${ }^{10,11}$.

Nicholson et al. propuseram uma classificação dos tumores de acordo com sua localização no saco herniário: intrasacular, quando o órgão com o tumor está encarcerado;

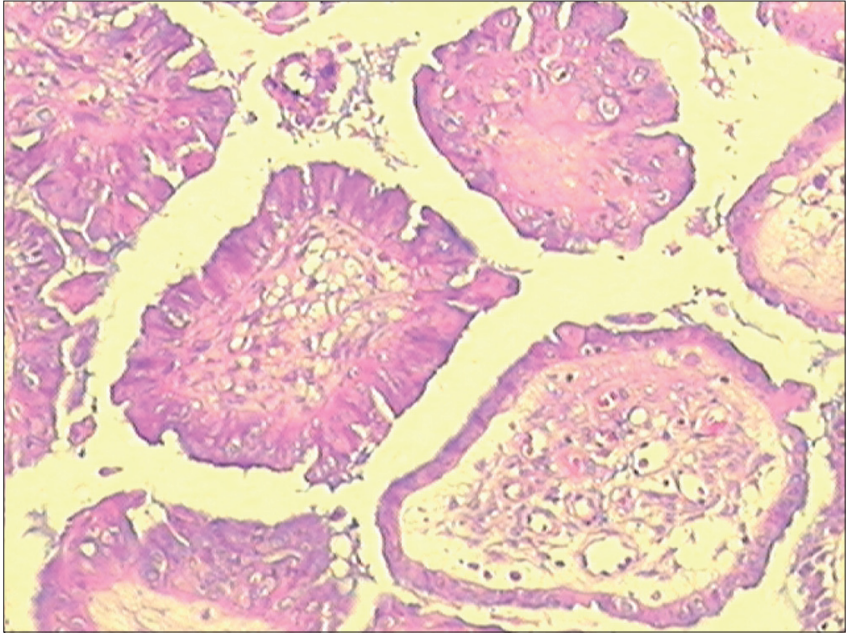

Figura 5 - Corte histológico de tumor borderline de ovário. Coloração hematoxilina-eosina. Aumento de 10x. Presença de múltiplas projeções papilíferas revestidas por epitélio formado por células colunares, com núcleos aumentados e vesiculares. Nota-se um aumento na relação nucleocitoplasmática e células indiferenciadas de citoplasma eosinofílico

sacular, quando o tumor está no interior do saco herniário ou com os elementos do cordão; extrasacular, quando o tumor localiza-se no interior do canal inguinal, mas não está no interior do saco herniário ${ }^{12}$.

Lesões ovarianas como conteúdo de hérnia inguinal manifestam-se mais comumente na infância, geralmente associadas a malformações genitourinárias ${ }^{13}$. Lesões malignas do ovário nessa topografia são extremamente raras, como, por exemplo, um caso descrito por Kawauchi et al. ${ }^{14}$, no Japão, em 1986, e um outro caso de disgerminoma bilateral descrito por Aksel'Dorf ${ }^{15}$. Outro relato evidenciou, durante uma hernioplastia laparoscópica, metástase de carcinoma ovariano, sendo a laparoscopia utilizada para se fazer o estadiamento da lesão ${ }^{16}$. Um caso na literatura inglesa de metástase na região inguinal de um tumor de ovário tratado foi identificado com o uso de PET scan, que pode auxiliar no diagnóstico e possibilita a análise de ressecção ${ }^{17}$.

Existem tumores mais raros, como angiomixoma, angiomiofibroblastoma, mesotelioma e até um colangiocarcinoma extra-hepático, que também foram relatados no interior do canal inguinal ${ }^{18-21}$

Em nosso caso, o exame intraoperatório de congelação foi negativo para malignidade. Entretanto, o laudo anatomopatológico final foi de tumor borderline. Esse tipo de tumor caracteriza-se pela proliferação epitelial atípica, sem invasão estromal ${ }^{22}$. Também é chamado de tumor de baixo grau de diferenciação maligna, carcinoma não-invasivo de baixo grau ou tumor de baixo potencial de malignidade. Representa de 15 a $20 \%$ dos tumores do ovário, e $81 \%$ das pacientes são classificadas como estádios I e II ${ }^{3,23}$. O pico de incidência ocorre em torno da quarta década de vida, faixa etária menor que as faixas em que ocorrem os tumores malignos, os quais têm maior 
incidência na quinta e sexta décadas ${ }^{24,25}$. Os tipos histológicos mais frequentes são o seroso e o mucinoso, e menos de $5 \%$ dos casos são tumores mistos, de células claras ou tumores de Brenner (células transicionais) ${ }^{26}$. São tumores de bom prognóstico com sobrevida global de 5 anos, que varia de 87,3 a $92,7 \%{ }^{3,24}$. Os níveis de CA-125 podem ser elevados nos tumores serosos, nos estádios avançados, na presença de ascite e em implantes peritoneais ${ }^{27}$.

$\mathrm{O}$ tratamento recomendado para as pacientes com prole constituída consiste de histerectomia total, salpingooforectomia bilateral, omentectomia infracólica, lavado peritoneal, biópsias randomizadas do peritônio, amostragem linfonodal paraórtica e pélvica e, nos tumores mucinosos, apendicectomia ${ }^{26,28}$. A amostragem linfonodal é questionada por alguns autores, já que a taxa de recorrência e sobrevida é semelhante entre as pacientes com linfonodos acometidos e não acometidos pelo tumor ${ }^{26,29}$.

Um fator limitante na decisão da extensão da cirurgia é o exame de congelação. A concordância do exame intraoperatório de congelação e análise patológica por hematoxilina-eosina é de, aproximadamente, $62 \%$ para os tumores borderline ${ }^{22}$, enquanto nos tumores epiteliais malignos esse índice de concordância pode chegar de 89 a $93 \%{ }^{30,31}$. A acurácia da congelação é menor nos tumores maiores que $20 \mathrm{~cm}$ e nos tumores mucinosos ${ }^{22,30}$. Em nosso caso, o tumor media, aproximadamente, $6 \mathrm{~cm}$ e a congelação mostrou-se negativa para malignidade, sendo o diagnóstico de tumor borderline confirmado pelo exame de hematoxilina-eosina.

O tratamento conservador pode ser realizado com segurança nas pacientes com tumores no estádio I e desejo gestacional. $\mathrm{O}$ tratamento defendido pela maioria dos autores é a ooforectomia unilateral com estadiamento completo. A retirada da lesão com preservação do ovário (cistectomia) está associada à maior chance de recidiva. Entretanto, se houver margens livres do tumor, esse procedimento é considerado seguro e factível nas pacientes que desejam preservar a fertilidade $e^{25,32}$. Nos casos de cirurgia conservadora, os tumores serosos mostraram-se como um fator independente de recidiva. A biópsia do ovário contralateral é controversa, pois cursa com infertilidade entre 10 a $20 \%$ dos $\operatorname{casos}^{25,26,32}$.

A laparoscopia é um método que vem sendo mais utilizado no diagnóstico e tratamento dos tumores borderline, embora a maioria dos estudos sejam retrospectivos e não-randomizados ${ }^{24,26}$. Embora não haja evidência de maior recorrência com o uso da laparoscopia ${ }^{24}$, pode ocorrer ruptura do tumor, o que é o fator prognóstico mais importante em relação à sobrevida livre de doença nos estádios iniciais ${ }^{33}$.

O acometimento extraovariano ocorre em até $30 \%$ dos casos de tumores boderline, sendo que a forma mais comum de apresentação é sob a forma de implantes peritoneais. Os tumores de arquitetura micropapilar apresentam comportamento mais agressivo, com maior incidência de implantes peritoneais invasivos ${ }^{28,34}$. Das pacientes que têm implantes peritoneais invasivos, a sobrevida é de $66 \%$ em 7 anos e de 95,3\% naquelas que não têm implantes ${ }^{28}$. A paciente descrita acima não apresentava lesões na cavidade abdominal e o lavado peritoneal foi negativo.

A cistectomia, níveis elevados de CA-125 e implantes invasivos são fatores prognósticos de recorrência nos tumores borderline do ovário ${ }^{24,25}$. No caso relatado, a paciente apresentava fatores de bom prognóstico: doença em estádio I, ausência de lesões no peritônio, CA-125 no limite da normalidade e submissão a tratamento conservador com ooforectomia.

Em função de a paciente apresentar insuficiência renal, idade avançada e fatores tumorais de bom prognóstico, optou-se por tratamento conservador, sem nova intervenção cirúrgica para histerectomia, omentectomia e biópsias peritoneais, prosseguindo com acompanhamento clínico por meio de exame ginecológico, ecografia abdominopélvica e dosagem sérica de CA-125.

\section{Referências}

1. Cannistra SA. Cancer of the ovary. N Engl J Med. 1993;329(21):1550-9

2. Heintz AP, Hacker NF, Lagasse LD. Epidemiology and etilogy of ovarian cancer: a review. Obstet Gynecol. 1985;66(1):127-35.

3. Heintz AP, Odicino F, Maisonneuve P, Quinn MA, Benedet JL, Creasman WT, et al. Carcinoma of the ovary. FIGO 6th Annual Report on the Results of Treatment in Gynecological Cancer. Int J Gynaecol Obstet. 2006;95 Suppl 1:S161-92 .

4. Flam F, Einhorn N, Sjövall K. Symptomatology of ovarian cancer. Eur J Obstet Gynecol Reprod Biol. 1988;27(1):53-7.

5. Leeming $R$, Olsen $M$, Ponsky JL. Inguinal dermoid cyst presenting as an incarcerated inguinal hernia. J Pediatr Surg. 1992;27(1):117-8.
6. Ryley DA, Moorman DW, Hecht JL, Alper MM. A mesothelial cyst of the round ligament presenting as an inguinal hernia after gonadotropin stimulation for in vitro fertilization. Fertil Steril. 2004;82(4):944-6

7. Rezkalla MA, Peterson KG, Ryan JJ. Pseudomyxoma peritonei: a case of mucinous adenocarcinoma of the appendix presenting as inguinal hernia. S D Med. 2006;59(2):54-5,57.

8. Meen $E$, Vergis $A$. Rare cause of an inguinal mass in pregnancy. Can J Surg. 2008;51(6):E124.

9. Ruiz-Tovar J, Ripalda E, Beni R, Nistal J, Monroy C, Carda P. Carcinoma of the sigmoid colon in an incarcerated inguinal hernia. Can J Surg. 2009;52(2):E31-2. 
10. Ben-Hur H, Schachter M, Mashiah A, Lifschitz-Mercer B, Pfeffermann $R$. Recurrent mucinous adenocarcinoma of the ovary presenting as an inguino-labial hernia. Eur J Gynaecol Oncol. 1996;17(4):299302.

11. Shimoyama S, Kuramoto S, Kawahara M, Yamasaki K, Endo $\mathrm{H}$, Murakami $\mathrm{T}$, et al. A rare case of pseudomyxoma peritonei presenting an unusual inguinal hernia and splenic metastasis. J Gastroenterol Hepatol. 2001;16(7):825-9.

12. Nicholson CP, Donohue JH, Thompson GB, Lewis JE. A study of metastatic cancer found during inguinal hernia repair. Cancer. 1992;69(12):3008-11

13. Ozkan OV, Semerci E, Aslan E, Ozkan S, Dolapcioglu K, Besirov E. A right sliding indirect inguinal hernia containing paraovarian cyst, fallopian tube, and ovary: a case report. Arch Gynecol Obstet. 2009;279(6):897-9.

14. Kawauchi N, Yashiro N, Ohtomo K, Itai $Y$, lio M, Furui S, et al. A case of ovarian tumor in inguinal hernia. Rinsho Hoshasen. 1986;31(3):441-3.

15. Aksel'Dorf AL. A case of bilateral disgerminoma of the ovary in bilateral inguinal hernia. Vopr Onkol. 1960;6(8):88-9.

16. Nair A, Vijay V, Francis D. An unsual inguinal "hernia"- the value of hernioscopic assessment. Surg Laparosc Endosc Percutan Tech. 2007; 17(4):349-50.

17. Díaz-Montes TP, Jacene HA, Wahl RL, Bristow RE. Combined FDG positron emission tomography and computed tomography for the detection of ovarian cancer recurrence in an inguinal hernia sac. Gynecol Oncol. 2005;98(3):510-2.

18. Minagawa T, Matsushita K, Shimada R, Takayama H, Hiraga R, Uehara $T$, et al. Aggressive angiomyxoma mimicking inguinal hernia in a man. Int J Clin Oncol. 2009;14(4):365-8.

19. Blel A, Mekni A, Bel Haj Salah M, Gargouri M, Azouz H, Haouet $S$, et al. Paratesticular angiomyofibroblastoma: case report and review of the literature. Pathologica. 2008;100(6):489-91.

20. Wheeler YY, Burroughs F, Li QK. Fine-needle aspiration of a well-differentiated papillary mesothelioma in the inguinal hernia sac: a case report and review of literature. Diagn Cytopathol. 2009;37(10):748-54

21. Yokoyama N, Shirai Y, Yamazaki H, Hatakeyama K. An inguinal hernia sac tumor of extrahepatic cholangiocarcinoma origin. World J Surg Oncol. 2006;4:13.

22. Kim JH, Kim TJ, Park YG, Lee SH, Lee CW, Song M, et al. Clinical analysis of intra-operative frozen section proven borderline tumors of the ovary. J Gynecol Oncol. 2009;20(3):176-80.
23. Tulpin L, Rouzier R, Morel O, Malartic C, Daraï E, Barranger E. Borderline ovarian tumors: an update. Gynecol Obstet Fertil. 2008;36(4):422-9.

24. Wu TI, Lee CL, Wu MY, Hsueh S, Huang KG, Yeh CJ, et al. Prognostic factors predicting recurrence in borderline ovarian tumors. Gynecol Oncol. 2009; 114(2):237-41.

25. Yokoyama Y, Moriya T, Takano T, Shoji T, Takahashi O, Nakahara K, et al. Clinical outcome and risk factors for recurrence in borderline ovarian tumours. Br J Cancer. 2006; 94(1 1):1586-91.

26. Tropé C, Davidson B, Paulsen T, Abeler VM, Kaern J. Diagnosis and treatment of borderline ovarian neoplasms "the state of the art"-. Eur J Gynaecol Oncol. 2009;30(5):471-82.

27. Lenhard MS, Nehring S, Nagel D, Mayr D, Kirschenhofer A, Hertlein L, et al. Predictive value of CA 125 and CA 72-4 in ovarian borderline tumors. Clin Chem Lab Med. $2009 ; 47(5): 537-42$.

28. Cadron I, Leunen K, Van Gorp T, Amant F, Neven P, Vergote I. Management of borderline ovarian neoplasms. J Clin Oncol. 2007;25(20):2928-37.

29. Seidman JD, Kurman RJ. Ovarian serous borderline tumors: a critical review of the literature with emphasis on prognostic indicators. Hum Pathol. 2000;31 (5):539-57.

30. Gorisek B, Stare MR, Krajnc I. Accuracy of intra-operative frozen section analysis of ovarian tumours. J Int Med Res. 2009;37(4): 1173-8

31. Yarandi F, Eftekhar Z, Izadi-Mood N, Shojaei H. Accuracy of intraoperative frozen section in the diagnosis of ovarian tumors. Aust N Z J Obstet Gynaecol. 2008;48(4):438-41.

32. Yinon Y, Beiner ME, Gotlieb WH, Korach Y, Perri T, Ben-Baruch G. Clinical outcome of cystectomy compared with unilateral salpingooophorectomy as fertility-sparing treatment of borderline ovarian tumors. Fertil Steril. 2007;88(2):479-84.

33. Vergote I, De Brabanter J, Fyles A, Bertelsen K, Einhorn N, Sevelda $\mathrm{P}$, et al. Prognostic importance of degree of differentiation and cyst rupture in stage I invasive epithelial ovarian carcinoma. Lancet. 2001;357(9251):176-82.

34. Prat J, De Nictolis $M$. Serous borderline tumors of the ovary: a long-term follow-up study of 137 cases, including 18 with a micropapillary pattern and 20 with microinvasion. Am J Surg Pathol. 2002;26(9): 1111 1-28. 